\title{
Breast ductal carcinoma in situ: a literature review of adjuvant hormonal therapy
}

\author{
Stephen Kinsey-Trotman, ${ }^{1}$ Zumin Shi, ${ }^{2}$ Beverley Fosh ${ }^{1}$ \\ ${ }^{1}$ Modbury Hospital, Modbury; ${ }^{2}$ The University of Adelaide, Adelaide, Australia
}

\begin{abstract}
This review paper analyzed publications of adjuvant tamoxifen and aromatase inhibitor use following surgery for breast ductal carcinoma in situ (DCIS). Key endpoint analyses were risk of invasive and noninvasive malignancies and new contralateral breast cancers. Metaanalysis of three studies showed a relative risk of 0.69 (95\% confidence interval, 0.60-0.79, $\mathrm{P}<0.05$ ) for breast malignancies with tamoxifen treatment in a mixed radiotherapy treatment/naïve cohort. Subgroup analysis of DCIS populations in multiple studies showed a trend to benefit with aromatase inhibitor treatment.
\end{abstract}

\section{Introduction}

Ductal carcinoma in situ (DCIS) is a non-invasive form of breast cancer accounting for twenty percent of mammogram-detected breast cancer in Western treatment centres. ${ }^{1}$ DCIS has been traditionally viewed as a precursor to invasive disease, however patients with DCIS have an increased risk of second breast malignancy and breast cancer death compared with the general population. ${ }^{2}$ Tamoxifen has become an established adjuvant treatment in the setting of oestrogen receptor positive invasive disease following definitive local treatment. Its role is

Correspondence: Stephen Kinsey-Trotman, Department of General Surgery Level 3, Room E111.3, Flinders Medical Centre, Flinders Drive, Bedford Park, South Australia 5042, Australia.

E-mail: kinstrot@gmail.com

Key words: Ductal carcinoma in situ; tamoxifen; aromatase inhibitor; breast cancer; hormonal; adjuvant.

Contributions: BF conceived the review and formulated the general design. SKT conducted literature review. Review articles chosen by SKT and BF. Analysis of articles completed by SKT with BF initial appraisal and feedback. Statistical analysis performed by ZS. Manuscript prepared by SKT. Manuscript reviewed by BF and ZS. All authors read and approved the final manuscript. SKT remained corresponding author for submission.

Received for publication: 18 July 2016.

Accepted for publication: 2 November 2016.

This work is licensed under a Creative Commons Attribution NonCommercial 4.0 License (CC BY-NC 4.0).

(C) Copyright S. Kinsey-Trotman et al., 2016

Licensee PAGEPress, Italy

Oncology Reviews 2016; 10:304

doi:10.4081/oncol.2016.304 less established as an adjuvant treatment for DCIS following breastconserving surgery, with or without radiotherapy. A limited number of studies have shown a statistically significant reduction in local DCIS recurrence with adjuvant tamoxifen therapy following surgical resection of oestrogen-receptor positive DCIS. Its application, however, remains limited by potential thromboembolic side effects and risk of endometrial cancer with extended therapy. ${ }^{3}$

The optimal management of DCIS, in particular adjuvant hormonal treatment following surgery, remains a vexing issue. Two seminal randomised studies reported the use of tamoxifen as adjuvant therapy in a population with DCIS treated with primary surgical excision, with or without radiotherapy. The incidence of local recurrence was reduced in the National Surgical Adjuvant Breast and Bowel Project (NSABP B24) trial, the findings, which were reported in $1999 .{ }^{4}$ These results were not supported by British data in the United Kingdom Coordinating Committee on Cancer Research (UKCCCR) trial where statistical significance was not reached. ${ }^{5}$ Outcomes from the above two trials were the subject of a Cochrane review which demonstrated a statistically signficicant reduction in non-invasive breast cancer events from pooled data with tamoxifen treatment following surgical excision. ${ }^{6}$

Aromatase inhibitors remain an established treatment in postmenopausal women with oestrogen-receptor positive invasive and metastatic disease. The drug class has a principle action in inhibiting the aromatase enzyme responsible for conversion of androgens synthesised in the adrenal medulla to oestrogen. Their role as an adjuvant treatment in DCIS remains controversial with limited studies published to date.

\section{Methods of research}

The primary clinical question: does adjuvant hormonal therapy in the form of tamoxifen or aromatase inhibitor treatment, following surgical excision of DCIS with or without radiotherapy reduce the risk of future breast malignancy, was the focus of our review. A secondary analysis based on local recurrence and contralateral breast cancer would also be posed.

To this end a review of the current literature was undertaken to source suitable published studies from peer-reviewed journals within the area.

A search of electronic databases 'MEDLINE' and 'PUBMED' for relevant published articles was undertaken in February 2015. Search terms were limited to those with accepted medical subject headings (MeSH) relevant to the clinical area and included: 'ductal carcinoma', 'DCIS', 'tamoxifen', 'aromatase inhibitor', 'breast cancer', 'breast neoplasm', 'hormonal' and 'adjuvant'. Publications deemed sufficiently relevant to the topic and published between January 1990 and February 2015 were included in the review. 
Publications for consideration for inclusion for review included randomised controlled trials, observational type studies and comparative studies. Articles regarding simple case reports, review articles or those limited to isolated in vitro research were not included in the review. Studies with patient cohorts with DCIS treated with tamoxifen or aromatase inhibitors from subgroup analysis or pooled data were also included in the review.

Where sufficient comparable trial data existed, a meta-analysis was undertaken using methods published by DerSimonian and Laird. ${ }^{7}$ Statistical analysis was undertaken using industry standard software such as Stata ${ }^{\circledR} 14$ or similar for combining risk estimate data. Tests for heterogeneity included q test statistic. Relative risk estimates and Forest Plot analysis were calculated for both primary and secondary clinical aims of the study. To maintain consistency in the statistical analysis, direct comparison of primary and secondary study outcomes was recalculated using the same statistical software. This resulted in small variances in relative risk estimates from those of the original publications.

\section{Results}

The preliminary literature review retrieved in excess of one hundred abstracts. Eliminating publications from animal and in vitro models and those articles without data involving adjuvant hormonal treatment or with specific reference to DCIS patient populations or subgroups, resulted in 31 publications remaining for further review. In this remaining selection were eight randomised-controlled trials (RCT), one cohort study and two comparative studies. The remaining articles were not included in the review: 13 review articles, two editorial or comment publications, two guidelines, one survey, and two articles which on subsequent review were found to be based on in vitro material and a case report.

Published results in one of the above eight randomised-controlled trials were not yet available and hence could not be included in the review, as was another RCT which published in vitro data. Neither of the two comparative studies reported results of adjuvant hormonal treatment for a DCIS population or subgroup and were eliminated from further review.

Further review of the literature in December 2015 using the search methods described above, did not yield any further relevant publications.

Two randomised-controlled trials and one cohort study with published outcome data on adjuvant tamoxifen treatment for DCIS postsurgery were included in the review. Five randomised-controlled trials of aromatase-inhibitors for DCIS post-surgery were included in the review.

\section{Tamoxifen}

A seminal study in the area of adjuvant therapy for DCIS was the NSABP B-24 study published in 1999. ${ }^{4}$ This was a multi-centre, double blind prospective randomised trial of 1804 women with DCIS. Both treatment arms were treated with wide local excision and fractionated radiotherapy (50 Gy) with randomised hormonal treatment to tamoxifen (20mg daily) or placebo. With a median follow-up period of 74 months, the study found fifty-three fewer breast cancers at 5 years in those treated with tamoxifen compared with placebo (11 vs $17 \%$, $\mathrm{P}=0.003$ ). There was also a reduction in new diagnoses of invasive ipsilateral malignancies in those treated with tamoxifen (RR $0.53,95 \% \mathrm{CI}$ $0.32-0.86, \mathrm{P}=0.01$ ). No change in overall survival was found between the two treatment groups. The study illustrated some limitations of tamoxifen therapy including thromboembolic events, which were higher in the tamoxifen treatment arm. The rate of endometrial cancer was higher in the tamoxifen group (7 cases, $0.12 \% / y e a r)$ versus placebo ( 3 cases, 0.06\%/year), $\mathrm{RR}=2.24$ (95\% CI, 0.51-13.44, $\mathrm{P}=0.38$ ).

The international UK/ANZ DCIS trial published initial results in 2003 and was followed by long-term results in $2011 .^{8}$ The cohort of 1701 women who underwent local excision for DCIS were randomised into fractionated radiotherapy at a total of 50Gy, tamoxifen (20 mg daily), or both. The mean follow-up was 12.7 years. Treatment with tamoxifen was demonstrated to reduce the rate of new ipsilateral DCIS with HR $0.70(0.51-0.86, \mathrm{P}=0.03)$ without a statistically significant reduction in ipsilateral invasive disease (HR 0.95, CI 0.66-1.38, $\mathrm{P}=0.79$ ). Tamoxifen treatment was also shown to reduce all new breast events compared to placebo HR 0.71 (95\% CI, 0.58-0.88, P=0.002). There was no statistically significant difference in mortality across all treatment groups. In addition, no statistically significant difference in treatment-related deaths (all cause and thromboembolic) were observed in the tamoxifen treatment arm compared to those not receiving hormonal treatment.

A recently published cohort study by the population-based Wisconsin in situ cohort study (WISC) group, investigated the disease-free survival (DFS) in a cohort of 1676 patients diagnosed with DCIS over a period of eleven years. ${ }^{9}$ The median age of women enrolled was 55.1 years and the majority were reported as post-menopausal (58.5\%). The mean follow-up period was 7.1 years (0.4-15.1 years). Total number of second breast cancer events was low for the population size at 143 documented cases. Tamoxifen uptake appeared to increase during the period of the study and a total of 358 patients were prescribed tamoxifen following breast conserving surgery and radiotherapy. In comparison, reported aromatase inhibitor use was low (42 patients following surgery and radiotherapy). Outcome data for patients treated with breast conserving surgery and adjuvant tamoxifen and radiotherapy showed a similar risk for a second cancer event as those women treated with ipsilateral mastectomy HR $=1.2,(95 \% \mathrm{CI}, 0.71-2.02)$.

\section{Statistical analysis}

Three studies were selected for meta-analysis; NSABP B-24, UK/ANZ and WISC. The NSABP B-24 trial cohort received radiotherapy following breast-conserving surgery and were divided into tamoxifen treatment and placebo cohorts. The UK/ANZ and WISC studies could be further subdivided into patients treated with tamoxifen both with and without radiotherapy. However, subgroup analysis of patients receiving tamoxifen and radiotherapy versus radiotherapy alone reduced overall treatment numbers markedly, meaning statistical significance was not reached in any new breast cancer risk category in these studies (Figures 1-4).

\section{All new breast malignancies}

All three of the above studies demonstrated a statistically significant risk reduction in all breast cancer recurrence events following treatment with breast conserving therapy and adjuvant tamoxifen treatment, in a combined radiotherapy and radiotherapy-naïve patient group (Figure 1; NSABP B-24 RR = 0.65, 95\% CI 0.52-0.83; UK/ANZ RR $=0.73,95 \%$ CI $0.61-0.88 ;$ WISC RR $=0.66,95 \%$ CI $0.45-0.98, \mathrm{P}<0.05$ ). The overall relative risk of the combined meta-analysis data for the three studies for all breast cancer recurrences was 0.69 (95\% CI, 0.60 $0.79, \mathrm{P}<0.005)$.

In the UK/ANZ trial, the only trend to risk reduction with tamoxifen in radiotherapy-treated patients was found in pooled 'all new' breast malignancies (RR 0.77; 95\% CI, 0.49-1.21, P<0.05) which were likely attributable to new DCIS cases (RR 0.69; 95\% CI, 0.34-1.4, P<0.05). The WISC study showed a risk reduction in all new breast cancers with tamoxifen treatment with prior radiotherapy (RR 0.62 ; 95\% CI, 0.39 $0.99, \mathrm{P}<0.05)$ although with low absolute numbers for new breast malignancies limiting further analysis. 


\section{New contralateral breast malignancies}

Analysis of contralateral breast cancer events in a combined radiotherapy and radiotherapy naïve patient group, in both the NSABP B-24 and UK/ANZ trials showed a statistically significant reduction in relative risk with hormonal therapy; $\mathrm{RR}=0.56(95 \% \mathrm{CI}, 0.34-0.90, \mathrm{P}<0.05)$ and $\mathrm{RR}=0.44(95 \% \mathrm{CI}, 0.25-0.77, \mathrm{P}<0.05)$, respectively (Figure 2). There was a trend to benefit in this patient group in the WISC study without reaching statistical significance $\mathrm{RR}=0.70$, (95\% CI, 0.36-1.37, $\mathrm{P}<0.05)$. Pooled study data gave an overall relative risk of new contralateral breast events of $0.54(95 \% \mathrm{CI}, 0.39-0.75, \mathrm{P}<0.05)$. The advantage of tamoxifen was lost when only those patients with prior radiotherapy treatment were included in the analysis, although absolute number of contralateral events were low (9/316).

\section{New non-invasive breast malignancies/ductal carci- noma in situ}

Only the UK/ANZ trial demonstrated a statistically significant reduction in non-invasive breast cancer events RR $=0.68$ (95\% CI, 0.52-0.90, $\mathrm{P}<0.05)$. The NSABP B-24 data showed a trend towards benefit for adjuvant treatment with tamoxifen in reducing new non-invasive breast malignancies, $\mathrm{RR}=0.76,(95 \% \mathrm{CI}, 0.53-1.08, \mathrm{P}<0.05)$ while no advantage was demonstrated in the WISC study, RR $=1.01$, (95\% CI, 0.48$2.08, \mathrm{P}<0.05)$. Combining the data through meta-analysis gave an overall relative risk of $0.73(95 \% \mathrm{CI}, 0.60-0.90, \mathrm{P}<0.05)$ for new non-invasive breast events (Figure 3). Limiting the analysis to those women who underwent previous treatment with radiotherapy did not significantly alter this trend, although lower patient numbers widened each corresponding confidence interval meaning statistical significance was no longer reached in the UK/ANZ group.
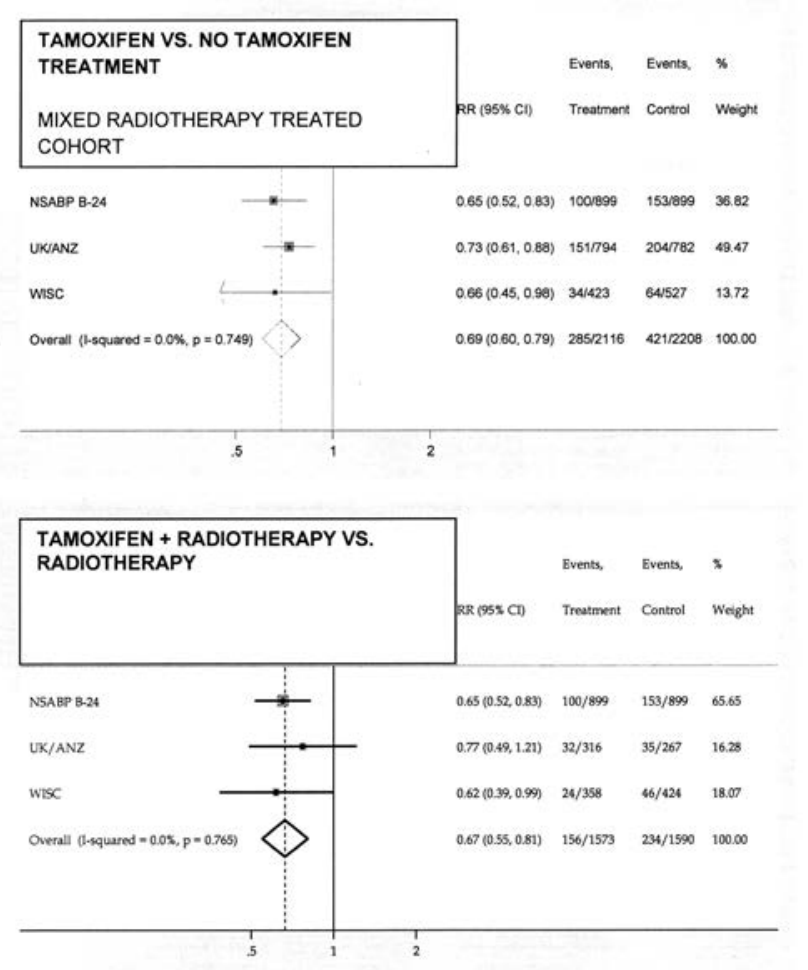

Figure 1. All new breast cancer events. First analysis includes radiotherapy treated and radiotherapy naïve patient cohort $+/$ tamoxifen. Second analysis includes only patients treated with adjuvant radiotherapy $+/$ - tamoxifen.

\section{New invasive breast malignancies}

The NSABP B-24 study demonstrated a statistically significant reduction in invasive breast cancer events following adjuvant tamoxifen therapy, RR $=0.57(95 \% \mathrm{CI}, 0.41-0.80)$. Both the UK/ANZ and WISC studies showed a trend towards benefit without reaching statistical significance for tamoxifen therapy in reducing invasive breast cancer post DCIS (RR $=0.80,95 \%$ CI, 0.59-1.08; RR $=0.50,95 \%$ CI $0.16-1.58$, $\mathrm{P}<0.05$ respectively). Combined meta-analysis of invasive breast cancer events from the three studies gave a relative risk of 0.68 (95\% CI, $0.54-0.84, \mathrm{P}<0.05)$, Figure 4. Limiting the analysis to those women in the UK/ANZ trial and WISC study that had previously undergone radiotherapy did no significantly alter this overall trend.

\section{Aromatase inhibitors}

The IBIS II was an international, double-blind, randomised placebocontrolled trial of 3864 postmenopausal women who were deemed at risk of breast cancer. ${ }^{10}$ The investigators used multiple criteria such as familial cancer history, individual cancer history and nulliparity prior to age 30 to determine those at higher risk of breast cancer. This also included a diagnosis of DCIS within the previous six months with completed local treatment, which represented about $8.3 \%$ and $8.5 \%$ in those allocated to the anastrozole and placebo treatment arms, respectively. Thirty-two women (2\%) in the anastrozole treatment group developed an invasive breast malignancy within the follow-up period of five years compared with 64 (3\%) women in the placebo group. This represented a hazards ratio of 0.50 for development of invasive breast cancer (95\% $\mathrm{CI}, 0.32-0.76, \mathrm{P}=0.001)$ and $\mathrm{HR}=0.47$ (95\% CI 0.32-0.68, $\mathrm{P}<0.0001)$ for any breast cancer. Unfortunately no subgroup analysis of DCIS patients was completed, limiting the applicability of the data to this
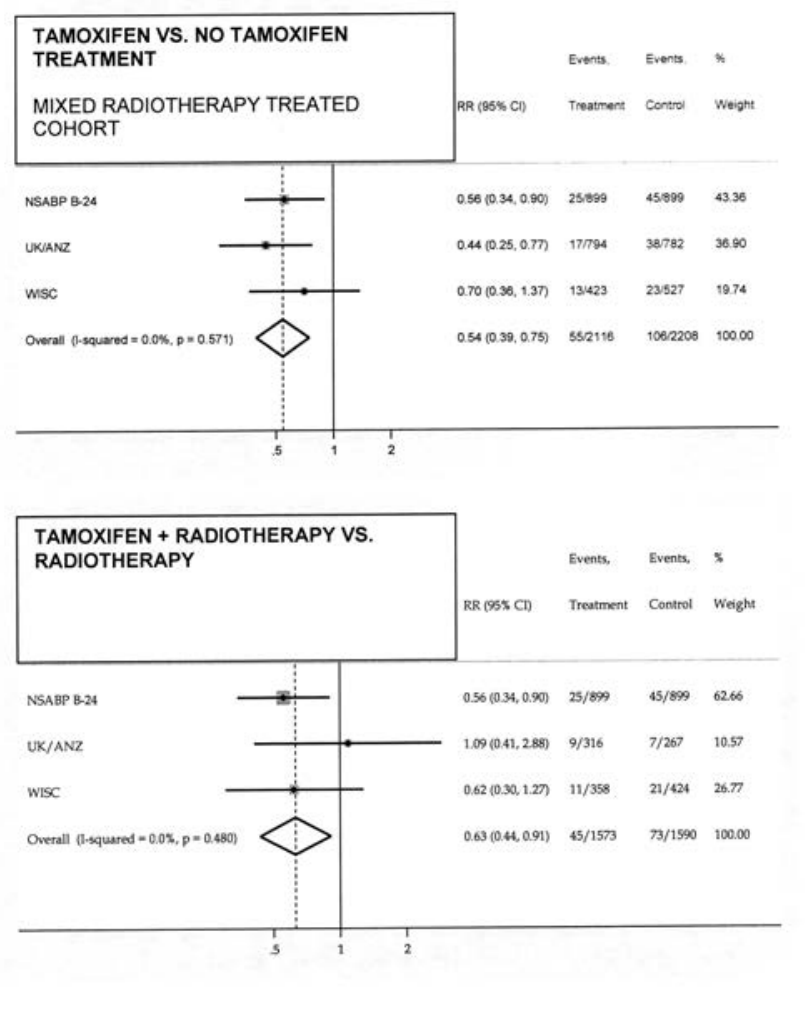

Figure 2. Contralateral new breast cancer events. First analysis includes radiotherapy treated and radiotherapy naïve patient cohort $+/$ - tamoxifen. Second analysis includes only patients treated with adjuvant radiotherapy $+/$ - tamoxifen. 
patient group. In the IBIS II trial the adverse effect profile of anastrozole showed a significant difference in musculoskeletal side effects such as moderate arthralgia, carpal tunnel syndrome and joint stiffness ( $64 \%$ versus $58 \%, \mathrm{RR}=1.1,95 \% \mathrm{CI} 1.05-1.16, \mathrm{P}<0.05$ ). There was also a higher report of vasomotor side effects and hypertension in the anastrozole treatment arm, without any overt statistically significant increases in thromboembolic events.

The National Cancer Institute of Cancer Clinical Trial Group (NCIC CTG) MAP.3 primary breast cancer prevention trial studied the effect of exemestane versus placebo in the prevention of invasive and non-invasive breast malignancies in 4560 post-menopausal women with a median follow-up interval of 35 months. ${ }^{11}$ This study population included about $2.5 \%$ participants who had previously been treated for DCIS with mastectomy. Eleven cases (0.4\%) of invasive breast cancer were reported in the exemestane treatment arm compared with 32 (1.4\%) for the placebo treated arm of the study, HR $=0.35$ (95\% CI, $0.18-0.70$, $\mathrm{P}=0.002)$. Total reported breast cancers were also less in the exemestane arm $(n=20,0.9 \%)$ compared to placebo $(n=44,2 \%), H R=0.47$ (95\% CI, $0.27-0.79, \mathrm{P}=0.004)$. Tolerability of exemestane was slightly less favourable to placebo with $15 \%$ discontinuing therapy secondary to side effects, compared with $10 \%$ for placebo. However, no statistically significant difference was found in fracture risk or cardiovascular events between the two treatment groups.

A number of trials have investigated the use of extended adjuvant treatment with aromatase inhibitors in postmenopausal women previously treated with five years tamoxifen therapy for early breast cancer. Trials of this extended adjuvant therapy include The National Cancer Institute of Canada Clinical Trials Group (NCIC CTG) MA.17 study of 5187 patients with randomisation to letrozole/placebo; NSABP B33 of
1598 patients randomised to exemestane/placebo and the Austrian Breast Cancer Study Group (ABCSG) 6A Trial of 856 patients randomised to anastrozole/placebo. ${ }^{12-14}$ All of these studies demonstrated a statistically significant reduction in breast cancer recurrence compared to placebo with extended adjuvant aromatase inhibitor therapy. However, the predominant patient population in these trials had undergone treatment for invasive breast cancers. In each trial no report was made on the number of study participants previously treated for DCIS.

\section{Statistical analysis}

Insufficient studies of aromatase inhibitor adjuvant treatment in DCIS following surgery were able to sourced. The above studies had limited subgroup data available for further statistical review. Metaanalysis was not completed for this treatment group.

\section{Discussion}

Combined data from studies of adjuvant hormonal treatment with tamoxifen post breast conserving surgery for DCIS have demonstrated a statistically significant reduction in new breast malignancies. The precise effect of radiotherapy in combination with tamoxifen is difficult to determine due to relatively low patient numbers in two trials selected for meta-analysis (UK/ANZ, WISC). The reduction in invasive ipsilateral malignancy in the NSABP B-24 study was not supported by data from UK/ANZ trial. Further, data from the UK/ANZ trial suggests that the reduction in new breast malignancies with tamoxifen therapy is largely attributable to non-invasive cancers. Previous authors have
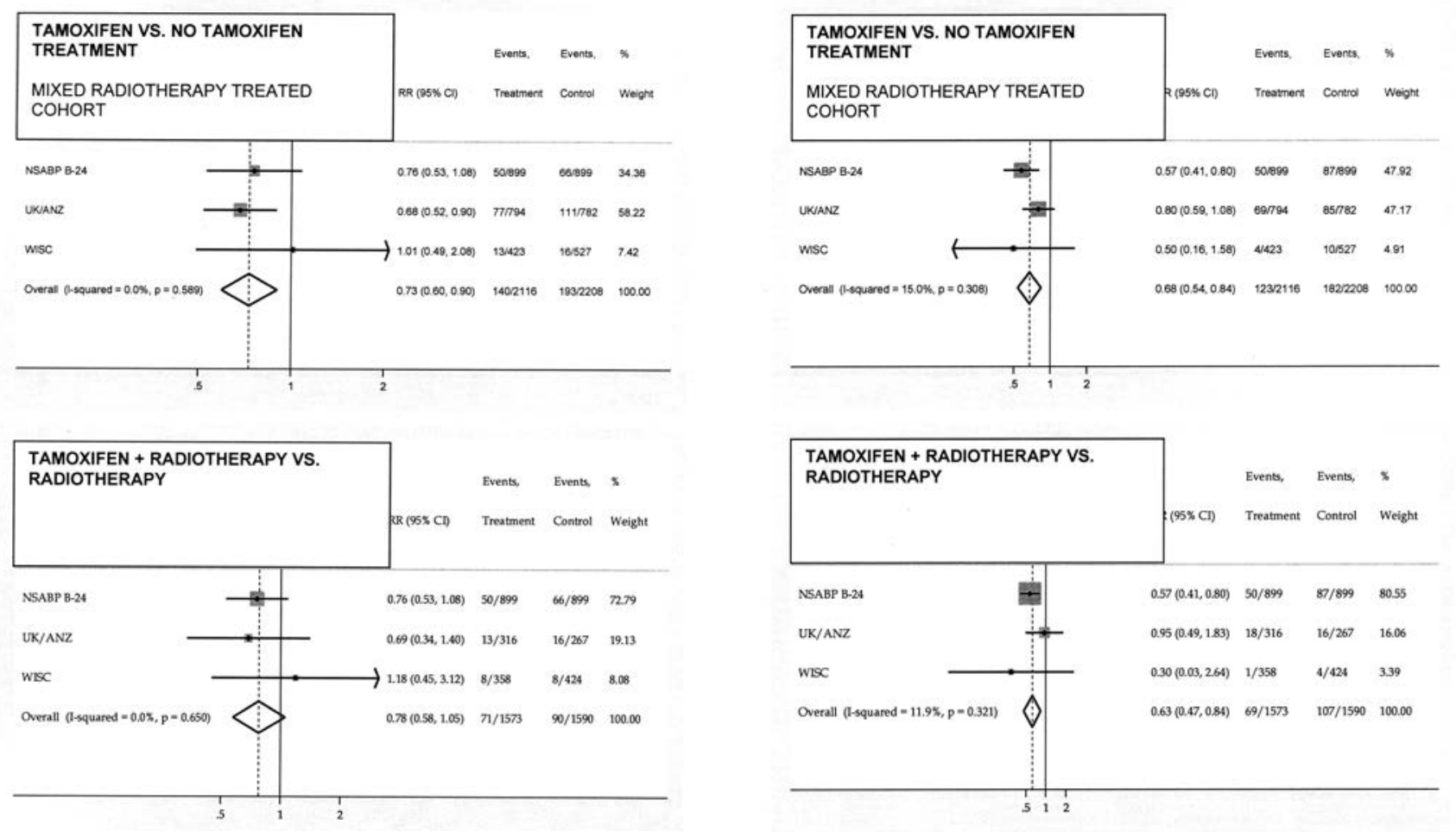

Figure 3. New non-invasive breast cancer/ductal carcinoma in situ events. First analysis includes radiotherapy treated and radiotherapy naive patient cohort $+/-$ tamoxifen. Second analysis includes only patients treated with adjuvant radiotherapy $+/$ tamoxifen.

Figure 4. New invasive breast cancer events. First analysis includes radiotherapy treated and radiotherapy naïve patient cohort $+/$ - tamoxifen. Second analysis includes only patients treated with adjuvant radiotherapy $+/$ - tamoxifen. 
proposed the younger age of the cohort in the American trial as well as the inclusion of patients with positive surgical resection margins for this discrepancy. ${ }^{6}$ In both trials a limitation of tamoxifen was its thromboembolic side effects, particularly limiting in older patient groups.

No analysis by outcome subgroup in the WISC study reached statistical significance, which is likely, a reflection of the low number of events recorded. This also limits further analysis by treatment group based on radiotherapy exposure.

The tolerability of tamoxifen remains an important limitation in its application. The associated risk of thromboembolic events and endometrial cancer risk are well documented with its use. ${ }^{5}$ It is perhaps this toxicity profile, combined with the broad utility of aromatase inhibitors as adjuvant treatment in the post-menopausal patient group that drives further study into their role for DCIS. Somewhat frustratingly, published randomised clinical trials utilising aromatase inhibitors as adjuvant treatment specifically to DCIS are lacking. Further, the side effect profile of aromatase inhibitors remains a limitation in some patients. The predominant side effects of this drug class include musculoskeletal type, vasomotor and bone mineral density loss, the former a having significant role in drug discontinuation in the above trials.

The IBIS II study demonstrated a reduction in DCIS and total breast cancers in a high-risk patient group treated with anastrozole. However, this heterogeneous group only had a minority of patients with a history of DCIS (around 8 percent) so results do not directly correlate with this patient subgroup.

Given DCIS remains a common pathological finding in resection specimens from health networks utilising mammogram screening programmes, further studies to determine optimum adjuvant hormonal therapy are needed. The large NSABP B-35 and IBIS II DCIS multicentre trials are notable. ${ }^{15,16}$ Each has reached accrual of a large postmenopausal DCIS cohort exceeding 3000 patients following breast conserving surgery with radiotherapy. Subgroup analysis of further therapy with tamoxifen and anastrozole should be instructive.

\section{Conclusions}

This review supports the role of tamoxifen in reducing the incidence of new breast malignancies post breast conserving surgery for DCIS, with or without radiotherapy. The utility of aromatase inhibitors in the adjuvant setting for DCIS will remain controversial until further clinical trials determine their role.

\footnotetext{
Abbreviations

ABCSG - Austrian Breast Cancer Study Group

$\mathrm{CI}$ - Confidence Interval

DCIS - Ductal Carcinoma In situ

DFS - Disease-Free Survival

IBIS - International Breast Cancer Intervention Studies

$\mathrm{MeSH}$ - Medical Subject Heading

NCIC CTG - National Cancer Institute of Canada Clinical Trials Group

NSABP - National Surgical Adjuvant Breast and Bowel Project

RCT - Randomised-Controlled Trial

UK/ANZ - United Kingdom and Australia New Zealand

UKCCCR - United Kingdom Coordinating Committee on Cancer Research

WISC - Wisconsin In situ Cohort
}

\section{References}

1. Zujewski JA, Harlan LC, Morrell DM, Stevens JL. Ductal carcinoma in situ: trends in treatment over time in the US. Breast Cancer Res Treat 2011;127:251-7.

2. Falk RS, Hofvind S, Skaane P, Haldorsen T. Second events following ductal carcinoma in situ of the breast: a register-based cohort study. Breast Cancer Res Treat 2011;129:929-38.

3. Skorafas GH, Farley DR, Peros G. Recent advances and current controversies in the management of DCIS of the breast. Cancer Treat Rev 2008;34:483-97.

4. Fisher B, Land S, Mamounas E, et al. Prevention of invasive breast cancer in women with ductal carcinoma in situ: an update of the national surgical adjuvant breast and bowel project experience. Semin Oncol 2001;28:400-18.

5. Houghton J, George WD, Cuzick J, et al. Radiotherapy and tamoxifen in women with completely excised ductal carcinoma in situ of the breast in the UK, Australia, and New Zealand: randomised controlled trial. Lancet 2003;362:95-102.

6. Staley H, McCallum I, Bruce J. Postoperative tamoxifen for ductal carcinoma in situ. Cochrane Database Syst Rev 2012;10:CD007847.

7. DerSimonian R, Laird N. Meta-analysis in clinical trials. Control Clin Trial 1986;7:177-88.

8. Cuzick J, Sestak I, Pinder S, et al. Effect of tamoxifen and radiotherapy in women with locally excised ductal carcinoma in situ: long-term results from the UK/ANZ trial. Lancet 2011;12:21-9.

9. Sprague, BL, McLaughlin, V, Hampton, JM, et al. Disease-free survival by treatment after a DCIS diagnosis in a population-based cohort study. Breast Cancer Res Treat 2012;141:145-54.

10. Cuzick J, Sestak I, Forbes JF, Dowsett M, et al. Anastrozole for prevention of breast cancer in high-risk postmenopausal women (IBIS-II): an international, double-blind, randomised placebo-controlled trial. Lancet 2014;383:1041-8.

11. Goss PE, Ingle JN, Ales-Martinez JE, et al. Exemestane for breastcancer prevention in postmenopausal women. $\mathrm{N}$ Engl $\mathrm{J}$ Med 2011;364:2381-91.

12. Ingle JN, Tu D, Pater JL, et al. Intent-to-treat analysis of the placebo-controlled trial of letrozole for extended adjuvant therapy in early breast cancer: NCIC CTG MA.17. Ann Oncol 2008;19:877-82.

13. Mamounas EP, Jeong JH, Wickerham DL, et al. Benefit from exemestane as extended adjuvant therapy after five years of adjuvant tamoxifen: intention-to-treat analysis of the national surgical adjuvant breast and bowel project b-33 trial. J Clin Oncol 2008;26:1965-71.

14. Jakesz R, Greil R, Gnant M, et al. Extended adjuvant therapy with anastrozole among postmenopausal breast cancer patients: results from the randomised Austrian breast and colorectal cancer study group trial 6a. J Natl Cancer Inst 2007;99:1845-53.

15. Julian TB, Land SR, Wolmark N. NSABP B-35: a clinical trial to compare anastrozole and tamoxifen for postmenopausal patients with ductal carcinoma in situ undergoing lumpectomy with radiation therapy. National surgical adjuvant breast and bowel project group: clinical trial protocol. Breast Dis 2003;14:121-2.

16. Cuzick J, Howell A, Forbes J. IBIS II: clinical trial protocol. Cancer Research UK; available from: http//www.ibis-trials.org/ Accessed: September 10, 2015. 